\title{
Large-scale confinement and small-scale clustering of floating particles in stratified turbulence
}

\author{
A. Sozza, ${ }^{1}$ F. De Lillo, ${ }^{1}$ S. Musacchio, ${ }^{2}$ and G. Boffetta ${ }^{1}$ \\ ${ }^{1}$ Department of Physics and INFN, Università di Torino, 1 Via P. Giuria, 10125 Torino, Italy \\ ${ }^{2}$ Laboratoire de Mathématiques J.A. Dieudomné, Université de Nice Sophia Antipolis, \\ CNRS, UMR No. 7351, 06100 Nice, France
}

(Received 12 January 2016; revised manuscript received 23 June 2016; published 6 September 2016)

\begin{abstract}
We derive a simple model, valid within the Boussinesq approximation, for the dynamics of small buoyant particles in stratified turbulence, in the presence of a mean linear density profile. By means of extensive direct numerical simulations, we investigate the statistical distribution of particles as a function of the two dimensionless parameters of the problem. We find that vertical confinement of particles is mainly ruled by the degree of stratification, with a weak dependence on the particle properties. In contrast, small-scale fractal clustering is found to depend on the particles relaxation time and is only slightly dependent on the flow stratification. The implications of our findings for the formation of thin phytoplankton layers are discussed.
\end{abstract}

DOI: 10.1103/PhysRevFluids.1.052401

Particles of density different from that of the surrounding fluid do not follow the motion of fluid particles and generate inhomogeneous distributions even in incompressible flows [1]. This phenomenon is crucial in a variety of instances, including cloud formation in the atmosphere, the dynamics of plankton in the ocean and lakes, and industrial applications [2-4]. The formation of inhomogeneous distributions in turbulent flows is also interesting from a theoretical point of view and, in recent years, analytical, numerical, and experimental studies have led to significant advances in the understanding of this process [5-10]. Most of the studies have considered the case of inertial particles that accumulate in regions of high vorticity (light particles) or high strain (heavy particles) $[5,7,11]$, as a consequence of the accelerations induced by the flow. Recent works have studied the interaction between gravity and turbulent accelerations in the dynamics of heavy particles. In particular, it has been shown that turbulence can increase their settling velocity with respect to still fluid, by pushing particles in regions of downward flow [12,13]. In the presence of density fluctuations, gravity also affects the flow itself as in the case of stratified turbulence, which finds many applications in natural flows [14,15], e.g., ocean dynamics in the presence of the pycnocline resulting from temperature and salinity variations [16,17]. Little is known about the distribution of floating particles in stratified turbulence, in spite of its relevance for applications. Recent works have studied the effect of stratification on the clustering of heavy [18] and light [19] particles, the formation of tangling clustering in stratified turbulence [20,21], and the effect of a vertical confinement in homogeneous turbulence [22].

One of the most remarkable examples of confinement of particles in the ocean is the formation of the so-called thin phytoplankton layers (TPLs): aggregations of phytoplankton and zooplankton at high concentration with thickness from centimeters to few meters, extending up to several kilometers horizontally and with a time scale from hours to days [23]. Among the possible mechanisms of formation of TPLs, convergence to a depth of neutral buoyancy has been proposed for nonswimming species and aggregates, such as diatoms and marine snow, which are often observed to accumulate in the presence of strong stratification [24].

In this work we investigate, by means of direct numerical simulations, the distribution of small buoyant particles transported in a turbulent stratified flow in the regime in which gravity dominates over the fluid acceleration. In this regime we consider a simple model for the particle motion that neglects inertial acceleration and balance the Stokes drag with the buoyancy forces. We study both the 
large-scale vertical distribution and the small-scale clustering of particles as a function of the relevant parameters. We find that small-scale (fractal) clustering is determined by the particles' relaxation time with a weak dependence on the degree of stratification. Conversely, the vertical confinement of particles is found to be mostly controlled by stratification. When rescaled to the typical parameters in the ocean, our results predict a fractal distribution for particles a few millimeters in size.

We consider a fluid linearly (and stably) stratified in the direction $z$ of gravity $\mathbf{g}=(0,0,-g)$ with a constant mean density gradient $d \rho / d z=-\gamma$. Within the Boussinesq approximation, the motion for the incompressible velocity field $\mathbf{u}(\mathbf{x}, t)$ is ruled by

$$
\begin{gathered}
\frac{\partial \mathbf{u}}{\partial t}+\mathbf{u} \cdot \nabla \mathbf{u}=-\frac{1}{\rho_{0}} \nabla p+v \nabla^{2} \mathbf{u}-N^{2} \theta \hat{\mathbf{z}}+\mathbf{f}, \\
\frac{\partial \theta}{\partial t}+\mathbf{u} \cdot \nabla \theta=\mathbf{u} \cdot \hat{\mathbf{z}}+\kappa \nabla^{2} \theta
\end{gathered}
$$

together with $\nabla \cdot \mathbf{u}=0$. The scalar field $\theta(\mathbf{x}, t)$, which in the above equations has the dimension of length, represents the deviations of the local density from the linear vertical profile $\rho=\rho_{0}-$ $\gamma(z-\theta)$. In addition, $v$ is the kinematic viscosity, $\kappa$ is the density diffusivity, and $N=\left(\gamma g / \rho_{0}\right)^{1 / 2}$ is the Brunt-Väisälä frequency. Furthermore, $\mathbf{f}$ represents an external mechanical forcing needed to sustain turbulence. In the inviscid unforced limit $(v=\kappa=\mathbf{f}=0)$ Eqs. (1) and (2) conserve the total energy, i.e., the sum of the kinetic and potential contributions $E=\frac{1}{2}\left\langle|\mathbf{u}|^{2}\right\rangle+\frac{1}{2} N^{2}\left\langle\theta^{2}\right\rangle$, where $\langle\cdots\rangle$ denotes the average over the domain.

The velocity $\mathbf{v}$ of a small inertial particle transported by the flow $\mathbf{u}$ generated by (1) evolves according to [25]

$$
\frac{d \mathbf{v}}{d t}=\beta \frac{d \mathbf{u}}{d t}-\frac{\mathbf{v}-\mathbf{u}}{\tau_{p}}+(1-\beta) \mathbf{g}
$$

where $\beta=3 \rho /\left(\rho+2 \rho_{p}\right)$ is the density ratio ( $\rho_{p}$ is the density of the particle of radius $a$ ) and $\tau_{p}=a^{2} / 3 v \beta$ is the viscous (Stokes) time. By introducing the covelocity $\mathbf{w}=\mathbf{v}-\mathbf{u}$ [7] we can rewrite (3) as

$$
\frac{d \mathbf{w}}{d t}=-(1-\beta) \frac{d \mathbf{u}}{d t}-\frac{\mathbf{w}}{\tau_{p}}+(1-\beta) \mathbf{g} .
$$

Without loss of generality, we set the particle density $\rho_{p}=\rho_{0}$ and, consistently with the Boussinesq approximation in Eqs. (1) and (2), we neglect the first term on the right-hand side of (4) [as $1-\beta \simeq\left(\rho_{0}-\rho\right) / \rho_{0}$ is a small parameter]. Moreover, having in mind the dynamics of plankton in the ocean, we have that typical turbulent accelerations are much smaller than $g$. We remark that additional terms (e.g., the Basset history term) are in general present in Eq. (3), but they would give a negligible contribution within the above approximation [26]. Considering now the limit of small particles, we obtain at the first order in $\tau_{p}$ an explicit expression for the velocity of the floater $\mathbf{v}$ whose position $\mathbf{x}$ evolves according to

$$
\frac{d \mathbf{x}}{d t}=\mathbf{v}=\mathbf{u}-\frac{1}{\tau}(z-\theta) \hat{\mathbf{z}},
$$

where we have used the fact that $(1-\beta) g \simeq \frac{2}{3} N^{2}(z-\theta)$ and we have introduced the particle relaxation time $\tau \equiv 3 / 2 N^{2} \tau_{p}$ to the isopycnal surface of density $\rho_{0}$. This surface, defined implicitly by the relation $z=\theta(x, y, z)$, will be denoted by $h(x, y)$, keeping in mind that in general it can be multivalued. The model (5) represents the simplest model for the dynamics of floaters in stratified flows and physically represents the balance between the Stokes drag and the buoyancy forces. A similar model has been developed and studied in Ref. [21]. Its validity is based on the assumptions of small density fluctuations (i.e., validity of the Boussinesq approximation) and small Stokes time of the particle. More general models, starting from the complete equation (3), can be considered, with the consequence of increased complexity and number of parameters [19]. 


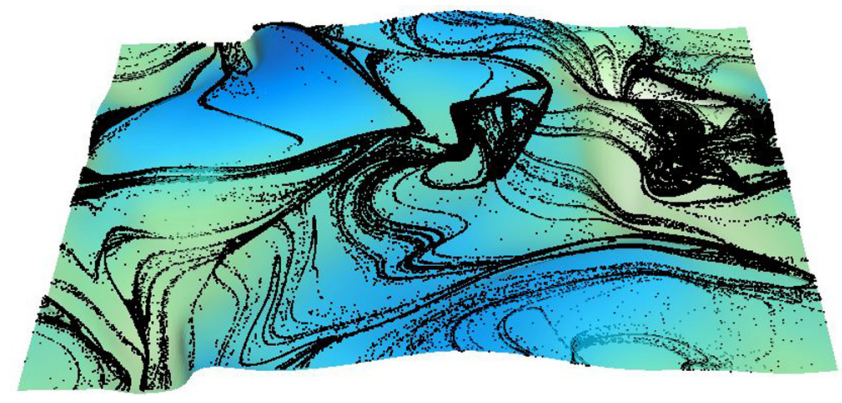

FIG. 1. Isopycnal surfaces $\theta=z$ corresponding to the density $\rho_{0}$ for a run at $\mathrm{Fr}=0.2$. The points represent the (fractal) distribution of buoyant particles with $\tau=0.1$ in the stationary state.

Although the fluid velocity $\mathbf{u}$ is incompressible, the velocity field transporting the floaters is not since $\boldsymbol{\nabla} \cdot \mathbf{v}=-(1-\partial \theta / \partial z) / \tau$, which is in general nonzero. This expression represents the rate of contraction of the phase space (here the configuration space) under the dynamics. When it is negative we expect that trajectories of floaters will collapse on a (dynamical) fractal attractor in the phase space. Examples of the attractor are displayed in Fig. 1, which shows that the large-scale confinement in the vertical direction coexists with a small-scale clustering with fractal distribution on the isopycnal surface.

We have integrated the Boussinesq equations (1) and (2) in a cubic domain of size $L=2 \pi$ with periodic boundary conditions by means of a fully parallel pseudospectral code at resolution up to $M=256$. Turbulence is generated by a $\delta$-correlated-in-time, isotropic forcing $\mathbf{f}$, which is active on a spherical shell of wave number around $k_{f}=2 \pi / L_{f}$ and pumps energy at the fixed rate $\varepsilon$. These parameters define, together with the Brunt-Väisälä frequency, the Froude number $\mathrm{Fr} \equiv \varepsilon^{1 / 3} k_{f}^{2 / 3} / N$, which measures the (inverse) stratification. Another relevant parameter in stratified turbulence is the buoyancy Reynolds number $\operatorname{Re}_{b}=\varepsilon / v N^{2}$ defined in terms of the ratio of the buoyancy (Ozmidov) scale $\ell_{B}=\varepsilon^{1 / 2} / N^{3 / 2}$ to the dissipative scale $\ell_{D}=v^{3 / 4} / \varepsilon^{1 / 4}$ as $\operatorname{Re}_{b}=\left(\ell_{B} / \ell_{D}\right)^{4 / 3}$, in analogy to the usual Reynolds number $\operatorname{Re}=\left(L_{f} / \ell_{D}\right)^{4 / 3}$. These three numbers are not independent since $\operatorname{Re}_{b} \propto \operatorname{Fr}^{2} \operatorname{Re}$ and $\operatorname{Re}_{b}=1$ discriminates between stratified-viscous flow $\left(\operatorname{Re}_{b}<1\right)$ and stratified turbulence $\left(\operatorname{Re}_{b}>1\right)$ [27]. Our simulations are mostly within the turbulent regime, with $\mathrm{Re}_{b}$ in the range $2 \leqslant \mathrm{Re}_{b} \leqslant 72$. We remark that in stratified turbulence the energy dissipation rate is in general different from the injection rate $\varepsilon$ as a fraction of the input is converted into potential energy [28]. In our simulations this difference is small and therefore, following the literature [27], we use the injection rate to define small-scale quantities.

Together with (1) and (2), we integrated Eq. (5) for the particle motion for a set of ten classes of floaters characterized by different values of $\tau$ in the range $0.1 \leqslant \tau \leqslant 10.0$. In presenting the results, this time will be made dimensionless with the Kolmogorov time $\tau_{D}=(v / \varepsilon)^{1 / 2}$ by introducing the relaxation number $R \equiv \tau / \tau_{D}$. We remark that $R$ is proportional to the inverse of the usual Stokes number St $\equiv \tau_{p} / \tau_{D}$, which is based on the particles' Stokes time $\tau_{p}$ [7]. Table I reports the most important parameters of the simulations.

TABLE I. Parameters of the simulations: $N$, resolution; $v$ and $\kappa$, kinematic viscosity and diffusivity, respectively; $L_{f}=2 \pi / k_{f}$, forcing scale; $\varepsilon$, energy input rate; $\ell_{D}=\left(v^{3} / \varepsilon\right)^{1 / 4}$, Kolmogorov scale; $\tau_{D}=$ $(\nu / \varepsilon)^{1 / 2}$, Kolmogorov time scale; $\operatorname{Re}=L_{f}^{4 / 3} \varepsilon^{1 / 3} / \nu ; \mathrm{Fr}=\varepsilon^{1 / 3} k_{f}^{2 / 3} / N$; and $R=\tau / \tau_{D}$. The actual range of $R$ depends on the value of Fr. All the simulations are performed at Schmidt number $\mathrm{Sc}=\nu / \kappa=1$.

\begin{tabular}{lcccccccc}
\hline \hline$M$ & $\nu, \kappa$ & $L_{f}$ & $\varepsilon$ & $\ell_{D}$ & $\tau_{D}$ & $\operatorname{Re}$ & Fr & $R$ \\
\hline 128 & $5 \times 10^{-3}$ & 3.72 & 0.195 & 0.028 & 0.16 & 363 & $0.2-1.0$ & $0.63-63$ \\
256 & $4 \times 10^{-3}$ & 3.72 & 0.195 & 0.024 & 0.14 & 454 & $0.2-1.0$ & $0.63-63$ \\
\hline \hline
\end{tabular}




\section{A. SOZZA, F. DE LILLO, S. MUSACCHIO, AND G. BOFFETTA}
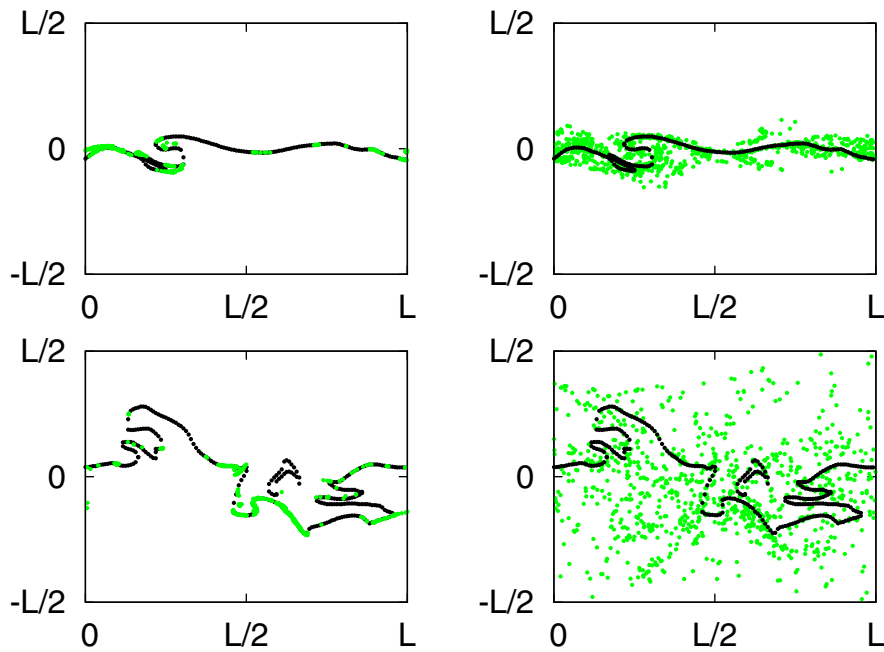

FIG. 2. Vertical $(x, z)$ sections of isopycnal surfaces $h$ (black points) for $\mathrm{Fr}=0.3$ (top row) and $\mathrm{Fr}=1.0$ (bottom row) together with the positions of buoyant particles (green points) with relaxation numbers $R=1.0$ (left column) and $R=60.0$ (right column).

In Fig. 2 we show vertical sections (at $y=0$ ) of the isopycnal surface $h$ [obtained from the solution of $z=\theta(x, y, z)]$ together with positions of the particles on the same sections, for different values of the parameters Fr and $\tau$. It is evident that the isopycnal surface $h$ is almost flat for strong stratification and it becomes more bent (and multivalued) as Fr increases. Figure 2 shows also the effect of the relaxation time $\tau$ on the particles. When $R=\tau / \tau_{D}<1$ the floaters are practically attached to the isopycnal surface, while their positions depart from the surface $h$ by increasing $\tau$.

The distribution of the vertical displacement $z$ of particles with respect to their equilibrium position in the absence of turbulence $z=0$ is found to be Gaussian in the wide range of parameters investigated. The standard deviations $\sigma_{z}$ of these vertical distributions of particles for different values of Fr and $R$ are shown in Fig. 3. We obtain a linear scaling of $\sigma_{z}$ on Fr, with a coefficient $\gamma$, which

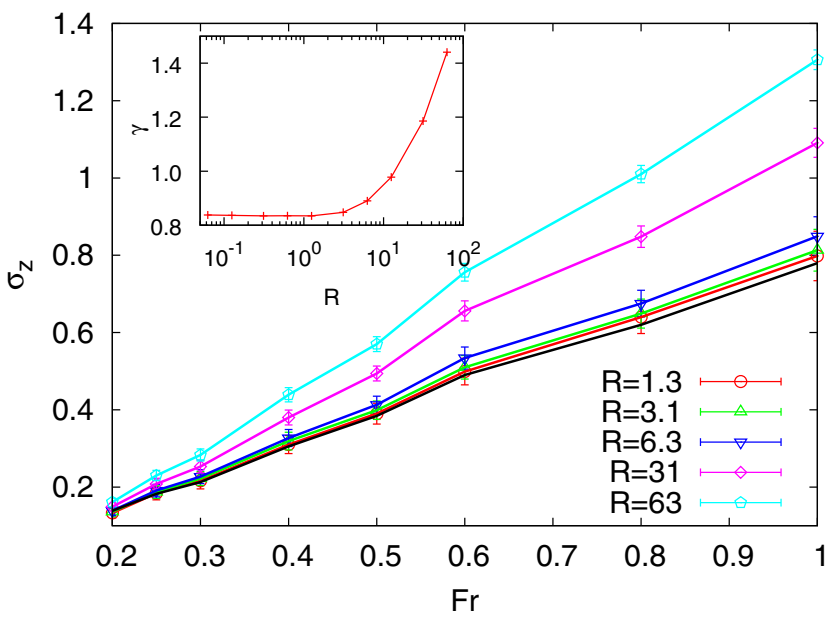

FIG. 3. Standard deviations $\sigma_{z}$ of the PDFs of particle vertical position as a function of Fr for different values of $R$ at $\operatorname{Re}=363$. The solid black line represents the standard deviation $\sigma_{h}$ of the isopycnal surface. The inset shows the slope $\gamma$ of the standard deviations obtained from the fit $\sigma_{z}=\sigma_{0}+\gamma \operatorname{Fr}$ as a function of $R$. 


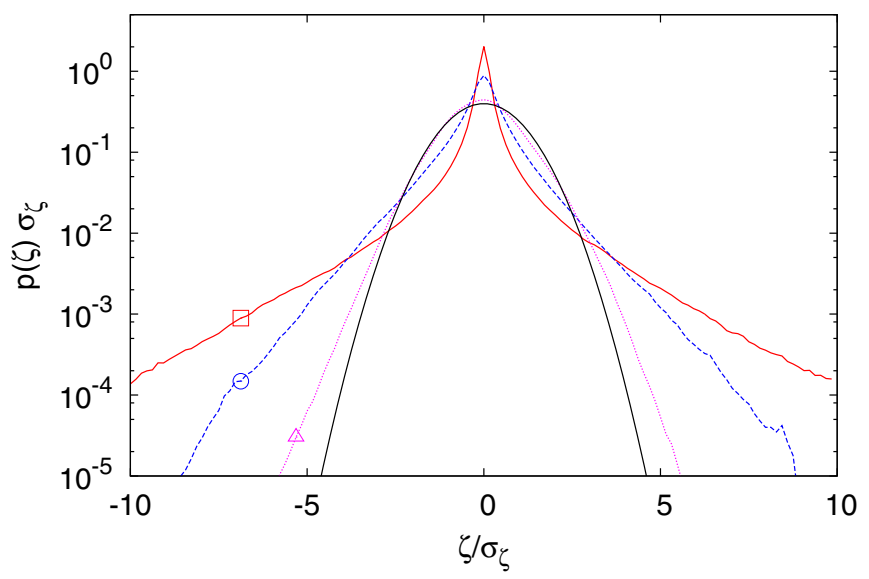

FIG. 4. Probability density functions of the variable $\zeta=z-\theta$ computed on particle positions for $\operatorname{Re}=454$, Fr $=1.0$, and $R=1.4$ (red line with square), $R=7.0$ (blue line with circle), and $R=70$ (pink line with triangle). The black line is a Gaussian distribution.

shows a (weak) dependence on $R$. For stronger stratification $\mathrm{Fr} \leqslant 0.3$, the standard deviation is almost independent of $R$ and the slope converges to $\gamma \simeq 0.84$, a feature that can be understood by looking at the plots in Fig. 2. The effect of the relaxation term in Eq. (5) is to allow the particle to detach from the level $z=\theta$ and to remain "suspended" for a time of order $\tau$ before experiencing the vertical velocity towards the isopycnal surface. Because in stratified turbulence the vertical velocity is suppressed [27], there is no mechanism that ejects particles far from the surface. Therefore, as shown in Fig. 2, the vertical region visited by particles (measured by $\sigma_{z}$ ) reflects the vertical extension of the isopycnal surface (given by its standard deviation $\sigma_{h}$ ), which, by definition, is independent of $\tau$. The linear dependence of $\sigma_{h}$ on Fr shown in Fig. 3 can be understood within the framework of stratified turbulence, as a manifestation of the presence of the so-called vertical shear layers [29] and the associated vertical correlation scale of velocity $L_{v}$. Physically, this scale represents the vertical displacement for converting injected kinetic energy into potential energy and can be estimated dimensionally as $L_{v} \simeq U / N$ ( $U$ is a typical large-scale velocity) and therefore one obtains $L_{v} \propto$ Fr, i.e., the linear scaling shown in Fig. 3 .

The deviation of particles from the isopycnal surface is studied by looking at the statistics of the variable $\zeta=z-\theta$ computed along particle trajectories. The dynamics of $\zeta$ is given by the time evolution of the field $\theta$ along the trajectory of a floater and gives (neglecting the diffusive term) $d(z-\theta) / d t=-(z-\theta)(1-\partial \theta / \partial z) / \tau$. In the absence of fluctuations $(\theta=0)$ this equation would simply represent the linear relaxation of particles towards the isopycnal layer $z=0$ [22]. This is not achieved since the term $\partial \theta / \partial z$ is fluctuating without a definite sign. Figure 4 shows the normalized probability density function (PDF) of the variable $\zeta$ for three different values of $\tau$ at $\mathrm{Fr}=1.0$. It is evident that the statistics is neither Gaussian nor scale invariant and the PDF develops large tails for small relaxation times [30]. These large fluctuations are due to the folding of the isopycnal surface. In correspondence with a fold, the stratification is inverted, $1-\partial \theta / \partial z$ changes sign, and $z-\theta$ grows exponentially (assuming that the field $\theta$ is quenched) until the particle reaches the nearest branch, above or below, of the isopycnal. This mechanism, which is enhanced at large Fr, produces large fluctuations of the distance between the particles and the isopycnal surface and causes the development of large tails in the PDF of $\zeta$.

As already discussed, floating particles moving according to (5) are transported by a compressible velocity field and are therefore expected to relax on a (dynamical) fractal subset of the physical space, as shown in the examples of Fig. 1. In order to characterize this subset and its dependence on the parameters, we have measured the correlation dimension $D_{2}$ of the particle distribution, defined as the scaling exponent of the probability of finding two particles at a distance less than $r$ : 


\section{A. SOZZA, F. DE LILLO, S. MUSACCHIO, AND G. BOFFETTA}

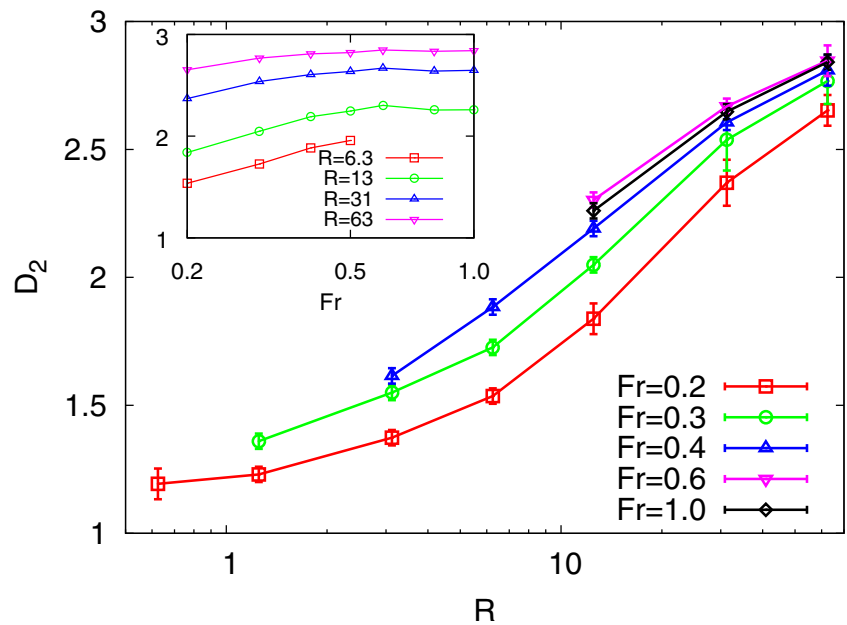

FIG. 5. Correlation dimension $D_{2}$ versus relaxation time $R$ for different values of the stratification parameter Fr. The error bars represent the fluctuation of the local scaling exponent in the fitting range of scales $(0.1 \eta \leqslant$ $r \leqslant 10 \eta$ ). The insert shows the correlation dimension $D_{2}$ plotted as a function of Fr for different values of $R$.

$P\left(\left|\mathbf{x}_{1}-\mathbf{x}_{2}\right|<r\right) \propto r^{D_{2}}$ as $r \rightarrow 0$ [31]. The maximum value $D_{2}=3$ denotes uniformly distributed particles, while $D_{2}<3$ indicates fractal patchiness, with smaller $D_{2}$ corresponding to more clustered distributions and increased probability of finding pairs of particles at close separation. Figure 5 shows $D_{2}$ as a function of $R$ for different values of Fr. As the model (5) has been derived under the assumption of small $\tau_{p} / \tau_{D}$, its validity is guaranteed above a minimum $R$ that depends on Fr. In Fig. 5 we plot the values of $D_{2}$ corresponding to the parameter space that satisfies the above condition.

In the limit $R \gg 1$, for which $\mathbf{v} \rightarrow \mathbf{u}$ in Eq. (5), floaters move as fluid particles in an incompressible velocity and therefore remain uniformly distributed in the volume with $D_{2}=3$. We remark that this limit is equivalent to taking $\tau_{p} \rightarrow 0$ in the general model of inertial particles (3). For smaller values of $R$ the fractal dimension decreases and reaches a value $D_{2} \sim 1$ for the stronger stratifications and the smallest relaxation time. This value indicates distributions of particles on quasi-one-dimensional structures (as shown qualitatively in Fig. 1).

The dependence of the fractal dimension on the degree of stratification is shown in the inset of Fig. 5. We observe that, in general, $D_{2}$ is virtually independent of $\mathrm{Fr}$ for $\mathrm{Fr} \geqslant 0.5$. This is in contrast with the (large-scale) vertical confinement shown in Fig. 4, which displays a strong (linear) dependence on Fr and a weak dependence on St.

In order to test the applicability of our results to the formation of TPLs we provide a simple example using the typical values observed for diatom-dominated marine snow [24]. Let us assume an energy dissipation rate $\varepsilon \simeq 10^{-8} \mathrm{~m}^{2} \mathrm{~s}^{-3}\left(\tau_{D} \simeq 10 \mathrm{~s}\right)$ and a Brunt-Väisälä frequency $N \simeq 0.1 \mathrm{~s}^{-1}$. The Froude number corresponding to $N \simeq 0.1 \mathrm{~s}^{-1}$ in our simulations is $\mathrm{Fr} \simeq 0.2$. At this Fr the thickness of the layer, measured by $\sigma_{z}$, is almost independent of the size of the particles (see Fig. 3). Conversely, the distribution of the particles within the layer can be very different. Aggregates of size $a \simeq 0.5 \mathrm{~cm}$ have a relaxation time $\tau \simeq 18 \mathrm{~s}$, which corresponds to $R=1.8$, at which we observe clustering at small scales with $D_{2}<2$ (Fig. 5). Smaller aggregates or single cells of size $a=0.1 \mathrm{~cm}$ correspond to $R \simeq 45$ and, according to our results, would distribute almost homogeneously (with a fractal dimension close to 3 ) within the thin layer. Our model allows estimating the characteristic time for the formation of TPLs. In particular, in the case of strong stratification in which the isopycnals are almost flat, our model predicts that the floaters converge exponentially toward the isopycnal $\rho=\rho_{p}$, with a relaxation time $\tau=3 / 2 N^{2} \tau_{p}$. Further field and laboratory experiments could provide evidence of this stratification-induced patchiness in thin phytoplankton layers. 


\section{LARGE-SCALE CONFINEMENT AND SMALL-SCALE ...}

In addition to the applications to thin layers, our results are of general interest as they describe quantitatively how the combination of turbulence and stratification generates both large-scale confinement and small-scale fractal patchiness in a suspension of buoyant particles.

This article is based upon work from European Cooperation in Science and Technology (COST) Action No. MP1305, supported by COST.

[1] M. W. Reeks, Transport, mixing and agglomeration of particles in turbulent flows, Flow Turbul. Combust. 92, 3 (2014).

[2] W. W. Grabowski and L. P. Wang, Growth of cloud droplets in a turbulent environment, Annu. Rev. Fluid Mech. 45, 293 (2013).

[3] S. Post and J. Abraham, Modeling the outcome of drop-drop collisions in diesel sprays, J. Multiphase Flow 28, 997 (2002).

[4] J. Seinfeld, Atmospheric Chemistry and Physics of Air Pollution (Wiley, New York, 1986).

[5] K. D. Squires and J. K. Eaton, Preferential concentration of particles by turbulence, Phys. Fluids A 3, 1169 (1991).

[6] E. Balkovsky, G. Falkovich, and A. Fouxon, Intermittent Distribution of Inertial Particles in Turbulent Flows, Phys. Rev. Lett. 86, 2790 (2001).

[7] J. Bec, Fractal clustering of inertial particles in random flows, Phys. Fluids 15, L81 (2003).

[8] G. Boffetta, F. De Lillo, and A. Gamba, Large scale inhomogeneity of inertial particles in turbulent flows, Phys. Fluids 16, L20 (2004).

[9] J. Bec, L. Biferale, M. Cencini, A. Lanotte, S. Musacchio, and F. Toschi, Heavy Particle Concentration in Turbulence at Dissipative and Inertial Scales, Phys. Rev. Lett. 98, 084502 (2007).

[10] I. Fouxon, Distribution of Particles and Bubbles in Turbulence at a Small Stokes Number, Phys. Rev. Lett. 108, 134502 (2012).

[11] L. P. Wang and M. R. Maxey, Settling velocity and concentration distribution of heavy particles in homogeneous isotropic turbulence, J. Fluid Mech. 256, 27 (1993).

[12] J. Bec, H. Homann, and S. S. Ray, Gravity-Driven Enhancement of Heavy Particle Clustering in Turbulent Flow, Phys. Rev. Lett. 112, 184501 (2014).

[13] K. Gustavsson, S. Vajedi, and B. Mehlig, Clustering of Particles Falling in a Turbulent Flow, Phys. Rev. Lett. 112, 214501 (2014).

[14] D. Lilly, Stratified turbulence and the mesoscale variability of the atmosphere, J. Atmos. Sci. 40, 749 (1983).

[15] J. Riley and M. Lelong, Fluid motions in the presence of strong stable stratification, Annu. Rev. Fluid Mech. 32, 613 (2000).

[16] S. A. Thorpe, An Introduction to Ocean Turbulence (Cambridge University Press, Cambridge, 2007).

[17] R. G. Williams and M. J. Follows, Ocean Dynamics and the Carbon Cycle (Cambridge University Press, Cambridge, 2011).

[18] M. van Aartrijk and H. J. H. Clercx, Preferential Concentration of Heavy Particles in Stably Stratified Turbulence, Phys. Rev. Lett. 100, 254501 (2008).

[19] M. van Aartrijk and H. J. H. Clercx, Vertical dispersion of light inertial particles in stably stratified turbulence: The influence of the Basset force, Phys. Fluids 22, 013301 (2010).

[20] A. Eidelman, T. Elperin, N. Kleeorin, B. Melnik, and I. Rogachevskii, Tangling clustering of inertial particles in stably stratified turbulence, Phys. Rev. E 81, 056313 (2010).

[21] T. Elperin, N. Kleeorin, M. Liberman, and I. Rogachevskii, Tangling clustering instability for small particles in temperature stratified turbulence, Phys. Fluids 25, 085104 (2013).

[22] M. De Pietro et al., Clustering of vertically constrained passive particles in homogeneous isotropic turbulence, Phys. Rev. E 91, 053002 (2015). 


\section{A. SOZZA, F. DE LILLO, S. MUSACCHIO, AND G. BOFFETTA}

[23] W. M. Durham and R. Stocker, Thin phytoplankton layers: Characteristics, mechanisms, and consequences, Annu. Rev. Marine Sci. 4, 177 (2012).

[24] A. L. Alldredge et al., Occurrence and mechanisms of formation of a dramatic thin layer of marine snow in a shallow Pacific fjord, Mar. Ecol. Prog. Ser. 233, 1 (2002).

[25] M. R. Maxey and J. J. Riley, Equation of motion for a small rigid sphere in a nonuniform flow, Phys. Fluids 26, 883 (1983).

[26] S. Olivieri, F. Picano, G. Sardina, D. Iudicone, and L. Brandt, The effect of the Basset history force on particle clustering in homogeneous and isotropic turbulence, Phys. Fluids 26, 041704 (2014).

[27] G. Brethouwer, P. Billant, E. Lindborg, and J. M. Chomaz, Scaling analysis and simulation of strongly stratified turbulent flows, J. Fluid Mech. 585, 343 (2007).

[28] A. Sozza, G. Boffetta, P. Muratore-Ginanneschi, and S. Musacchio, Dimensional transition of energy cascades in stably stratified forced thin fluid layers, Phys. Fluids 27, 035112 (2015).

[29] P. Billant and J. M. Chomaz, Self-similarity of strongly stratified inviscid flows, Phys. Fluids 13, 1645 (2001).

[30] D. A. Birch, W. R. Young, and P. J. S. Franks, Plankton layer profiles as determined by shearing, sinking, and swimming, Limnol. Oceanogr. 54, 397 (2009).

[31] G. Paladin and A. Vulpiani, Anomalous scaling laws in multifractal objects, Phys. Rep. 156, 147 (1987). 